\title{
Sistema cannabinoide y regulación de la conducta alimentaria
}

\author{
Francisco Arias Horcajadas* \\ *Unidad de Psiquiatría. Fundación Hospital Alcorcón. Madrid. \\ Enviar correspondencia a: \\ Francisco Arias Horcajadas. Unidad de Psiquiatría. Fundación Hospital Alcorcón. \\ 28922 Alcorcón (Madrid). E-mail: farias@fhalcorcon.es
}

Recibido: Septiembre de 2006 Aceptado: Octubre de 2007

\section{RESUMEN}

Cada vez disponemos de más información que sugiere que el sistema cannabinoide es un mecanismo crucial en la regulación de la ingesta alimentaria y en el metabolismo energético. De ahí que próximamente se vaya a comercializar un antagonista cannabinoide, el rimonabant, para su uso en obesidad que, además de una pérdida de peso, consigue una mejora del denominado síndrome metabólico, con unos cambios en el metabolismo lipídico y glucídico no observados por otros fármacos antiobesidad actualmente comercializados. Se presenta una revisión de los conocimientos actuales sobre el tema y los datos de estudios propios: estudios genéticos de dicho sistema en los trastornos de la conducta alimentaria y en obesidad y estudios de localización de receptores cannabinoides en sitios relacionados con la ingesta. Dichos estudios apoyan un estado de hiperactividad cannabinoide en la obesidad y, además, dicha hiperactividad puede constituir un factor pronóstico.

Palabras clave: sistema cannabinoide, obesidad, trastornos de la alimentación, ingesta, metabolismo energético.

\begin{abstract}
There is increasing evidence to suggest that the cannabinoid system is a crucial mechanism in the regulation of feeding and metabolism. It is against this background that a cannabinoid antagonist, rimonabant, is about to come onto the market for the treatment of obesity. Moreover, in addition to weight-loss effect, this drug has a beneficial effect on the so-called metabolic syndrome, with changes in the lipid and glucidic metabolism not observed for other anti-obesity drugs currently available. We present a review of current knowledge in this field and data from our own studies: genetic studies of this system in eating disorders and in obesity and studies of localization of cannabinoid receptors at sites related to feeding. These studies support a state of cannabinoid hyperactivity in obesity; furthermore, such hyperactivity can constitute a prognostic factor.
\end{abstract}

Key words:cannabinoid system, obesity, eating disorders, feeding, metabolism.

\section{INTRODUCCIÓN*}

E cannabis fue usado con fines medicinales en China 4.000 años a.C. Desde allí, la planta se extendió por India y el Sudeste Asiático, llegando a la civilización grecorromana. En la India, en el siglo XV a.C., se usaba el cáñamo por su efecto estimulante del apetito (Grinspoon y Bakalar, 1993). Durante la Edad Media, los árabes (Al-Badri, 1251 d.C.) recomendaron el uso médico del cannabis por esa propiedad. En 1842, el empleo del cannabis como remedio medicinal fue introducido en Gran Bretaña con el regreso de un cirujano irlandés que había estado en la India, O’Shaughnessy. Este médico hizo

\footnotetext{
* INDICE DE ABREVIATURAS (según orden de aparición en el texto): SEC: sistema cannabinoide. VMH: núcleo ventromedial hipotalámico. LH: Núcleo lateral del hipotálamo. ARC: núcleo arcuato hipotalámico. PVN: Núcleo periventricular. DMH: núcleo dorsomedial hipotalámico. NAc: Núcleo accumbens. NTS: Núcleo del tracto solitario. BHE: barrera hematoencefálica. NPY: neuropéptido Y. AgRP: péptido relacionado con el agouti. Alfa-MSH: hormona estimulante de melanocitos. CART: transcripto regulado por anfetamina y cocaína. CCK: colecistokinina. PYY: péptido YY. DIO: obesidad inducida por la dieta. KO: knock-out. THC: tetrahidrocannabinol. AEA: anandamida. 2AG: 2 araquidonilglicerol. POMC: proopiomelanocortina. VTA: área tegmental ventral. OEA: oleiletanolamina. CB1: receptor cannabinoide tipo 1. FAAH: amidohidrolasa de ácidos grasos. FAS: sintasa de ácidos grasos. GLP1: péptido similar al glucagon tipo 1. TCA. Trastornos de la conducta alimentaria. IMC: ïndice de masa corporal. RIO: ensayos clínicos de rimonabant en obesidad. STRATUS: estudios con rimonabant en el uso de tabaco. HDL: lipoproteína de alta densidad.
} 
popular la utilización de la denominada tintura de cannabis para distintos estados patológicos, describiendo su efecto sobre el apetito (Ramos y Fernández, 2000). En una publicación en la revista Lancet en 1889, Birch refirió el empleo del cannabis para la dependencia de opiáceos, observando dicho incremento del apetito. En 1942, la revista JAMA en una editorial, reconoce la pérdida de apetito como un potencial uso terapéutico para el cannabis.

Los consumidores de cannabis describen un incremento pertinaz del apetito, incluso si están saciados previamente. Con frecuencia, se refiere una mayor apetencia por los dulces (Hollister, 2001).

El interés del tema ha resurgido a raíz de la descripción del sistema endocannabinoide (SEC) y la síntesis de agonistas y antagonistas de este, produciéndose una gran proliferación de trabajos en experimentación animal que van constatando la relevancia del SEC en la regulación del apetito, aunque sea prematuro aún valorar las implicaciones clínicas y terapéuticas.

\section{Neuroanatomía básica de la regulación de la con- ducta alimentaria}

A partir de una serie de estudios experimentales en animales llevados a cabo en las décadas de los años 40 y 50 provocándoles lesiones cerebrales localizadas, se implantó la hipótesis dual hipotalámica que ha estado vigente hasta muy recientemente. Según esta hipótesis existiría un centro de la saciedad (núcleo ventromedial hipotalámico, VMH) y un centro del hambre (núcleo lateral hipotalámico, LH) (Stellar, 1954). En la actualidad, se acepta que la regulación es más compleja e intervienen otros centros neurales con una relevancia similar, es decir, se pasa de una estructura jerárquica a la distribución de centros cerebrales de rango similar distribuidos en troncoencéfalo, sistema límbico e hipotálamo. Así hay que destacar una serie de estructuras hipotalámicas como son los núcleos arcuato (ARC), VMH, LH, paraventricular (PVN), dorsomedial (DMH); además de otros núcleos como el núcleo accumbens (NAc) que forma parte de los circuitos de recompensa cerebral, el núcleo del tracto solitario (NTS) y núcleo parabraquial del troncoencéfalo y diversas estructuras del cortex cerebral (Broberger, 2005; Berthoud, 2002).

El núcleo arcuato (ARC) es fundamental. Esté núcleo se encuentra próximo a la eminencia media, donde a través de la mayor permeabilidad de la barrera hematoencefálica $(\mathrm{BHE})$ recibe información directa del torrente circulatorio (por ejemplo estado de la glucemia). A este núcleo le llega información del estado de los depósitos energéticos a través de hormonas como la leptina y la insulina que indican las reservas del tejido adiposo y la grelina que informa sobre el estado del tubo digestivo. En este centro existen dos grupos neuronales con una serie de neuropéptidos relevantes en la regulación de la conducta alimentaria como el neuropéptido Y (NPY) y la proteína relacionada con el agouti (AgRP) de efecto orexígeno y la hormona estimulante de melanocitos ( $\alpha \mathrm{MSH}$ ) y el transcripto regulado por anfetaminas y cocaína (CART) de efecto anorexígeno (Wynne, Stanley, McGowan y Bloom, 2005).

Los núcleos hipotálamicos conectan con el núcleo accumbens (NAc), interaccionando de esta forma los sistemas de regulación de la conducta alimentaria con los de recompensa cerebral. A su vez, ambos centros presentan aferencias y eferencias con el núcleo del tracto solitario (NTS) del troncoencefálo que recibe la información fundamental de la periferia a través del nervio vago y a través de la sangre por la cercanía con el área postrema que es otra zona de mayor permeabilidad de la BHE (Wynne et al. 2005).

Además de la regulación central, existe un complejo mecanismo de señales periféricas que regulan la ingesta y el metabolismo a corto y largo plazo. Hay que destacar el papel de las hormonas ya mencionadas leptina e insulina que informan sobre los depósitos grasos y la información procedente del tubo digestivo a través de dos vías, humoral y nerviosa. La grelina se libera en el estómago e intestino en situaciones de ayuno e informa al núcleo ARC vía humoral y a través del nervio vago. Es la primera hormona descrita del sistema digestivo que estimula la ingesta. La colecistokinina (CCK) liberada en el estómago es una de las principales señales de saciedad a corto plazo. El péptido YY (PYY) igualmente tiene efecto saciante, siendo liberado en íleon y colon. El nervio vago transmite a los núcleos dorsales del vago y al NTS información de señales físicas (distensión gástrica) y químicas (CCK). El tejido adiposo, que durante mucho tiempo se consideraba un órgano inerte, se va constituyendo como uno de los principales órganos endocrinos del organismo, siendo el responsable de secretar un amplio número de hormonas. Además de la leptina, va adquiriendo importancia el papel de la adiponectina, sustancia que se secreta según el estado de los depósitos grasos y que tiene un papel fundamental favoreciendo la lipolísis (Broberger, 2005).

Pues bien, cada vez disponemos de más datos que indican que el sistema cannabinoide (SEC) tiene un papel fundamental en la regulación de la ingesta alimentaria y el metabolismo energético. Para revisar dicho papel vamos a hacer referencia a tres aspectos: Estudios procedentes de experimentación animal, estudios en humanos y descripción de los mecanismos centrales y periféricos. 


\section{Los agonistas cannabinoides producen incremento del apetito y peso en experimentación animal}

Una serie de investigaciones se han centrado en el estudio del papel del SEC en la regulación del apetito, el peso y el balance energético en animales, apoyando un efecto estimulante del apetito de los agonistas cannabinoides y un efecto anorexígeno de los antagonistas. El uso de diversos modelos experimentales ha ocasionado algunos resultados discrepantes. Se han usado modelos de animales deprivados de alimentos desde 1 hora hasta 24 horas, posteriormente presaciados antes de la administración de la sustancia experimental o no presaciados (modelo de animales hiperfágicos en este último caso), y podían estar deprivados de agua o no simultáneamente. Otros autores han empleado modelos de animales con libre acceso a la comida, con dietas ricas en hidratos de carbono, ricas en grasas o dietas estándar. Por otro lado, son interesantes los datos aportados por los modelos animales de obesidad, ya sean inducidos por dieta (DIO) o manipulados genéticamente (modelos de ratones ob/ob o db/db, ya sean con déficit del gen de la leptina o déficit del receptor de esta respectivamente, modelo de ratas obesas Zucker, etc). Por último, son muy útiles los modelos de animales knock-out (KO) para el receptor CB1, es decir, carentes de este.

Los estudios iniciales con agonistas cannabinoides describían un efecto hipofágico pero fue debido al uso de dosis elevadas, predominando el efecto sedante. Los experimentos más recientes confirman el efecto orexígeno de los agonistas incluso en animales saciados y dicho efecto está mediado por los receptores CB1. Los agonistas cannabinoides más usados son el $\triangle^{9}$-Tetrahidrocannabinol (THC) y un agonista endógeno como la anandamida (AEA) que aumentan la ingesta en distintos procedimientos experimentales (Hao, Avraham, Mechoulam y Berry, 2000; MartínezGonzález, Bonilla, Morales, Henriksen, Velazquez y Próspero, 2004; Wiley, Burston, Leggett, Alekseeva, Razdan, Mahadevan et al. 2005; Williams y Kirkham, 1999; Williams y Kirkham, 2002; Williams, Rogers y Kirkham, 1998). Otros agonistas como el 2-araquidoniIglicerol (2-AG) (Kirkham, Williams, Fezza y Di Marzo, 2002) y el noladin eter (Avraham, Menachem, Okun, Zlotarav, Abel, Mechoulam et al. 2005) producen un efecto similar. Recientemente se ha descrito que el $\Delta^{8}$ $\mathrm{THC}$, con menos efectos psicoactivos que el $\Delta^{9} \mathrm{THC}$, podría tener un efecto orexígeno más potente (Avraham, Ben-Shushan, Breuer, Zolotarev, Okon, Fink et al. 2004).

El efecto orexígeno se observa con la administración de agonistas en centros cerebrales relacionados con la regulación de la ingesta, como el núcleo VMH (Jamshidi y Taylor, 2001); en el núcleo accumbens (NAc) (Kirkham et al. 2002) o en el cuarto ventrícu- lo por la proximidad del núcleo parabraquial (Miller, Murray, Freeman y Edwards, 2004).

\section{Los antagonistas cannabinoides en administración central y periférica disminuyen la ingesta y el peso en animales}

La administración de un antagonista cannabinoide como el rimonabant a dosis de $1-10 \mathrm{mg} / \mathrm{kg}$ en experimentos de 14 a 35 días, disminuye la ingesta en animales con acceso ilimitado a la comida (Colombo, Agabio, Diaz, Lobina, Reali y Gessa, 1998; Freedland, Poston y Porrino, 2000; Simiand, Keane, Keane y Soubrie, 1998) y en animales deprivados de alimentos (Gómez, Navarro, Ferrer, Trigo, Bilbao, Del Arco et al. 2002; Rowland, Mukherjee y Robertson, 2001). El mismo efecto se ha observado con otros antagonistas cannabinoides en administración central (Koch y Werner, 2000). La pérdida de peso oscila alrededor del 20\% del peso basal (Pagotto y Pasquali, 2006).

Existe controversia sobre sí el efecto anorexígeno es preferentemente sobre algún tipo de alimentos. En una serie de experimentos se observa un efecto preferencial sobre la ingesta de alimentos más apetitosos (Arnone, Maruani, Chaperon, Thiebot, Poncelet, Soubrie et al. 1997; Simiand et al. 1998, Ward y Dykstra, 2005), mientras que otros experimentos indican que los antagonistas diminuyen la ingesta de dietas ricas en hidratos de carbono, ricas en grasas y dietas estándar tanto en animales deprivados como no de alimentos (Freedland et al. 2000; McLaughlin, Winston, Swezey, Wisniecki, Aberman y Tardif et al. 2003; Verty, McGregor y Mallet, 2004; Rowland et al. 2001).

\section{Existe tolerancia a los efectos anorexigenos de los antagonistas cannabinoides pero no a sus efectos metabólicos y hay un efecto rebote tras la supresión}

En los experimentos con animales con antagonistas cannabinoides, fundamentalmente el rimonabant, se ha objetivado que a los 4-5 días de la administración se producía tolerancia a sus efectos anorexígenos, aunque el descenso del peso se mantenía durante el resto del experimento (Bensaid, GaryBobo, Esclangon, Maffrand, Le Fur, Oury-Donat et al. 2003; Colombo et al. 1998, Ravinet Trillou, Arnone, Delgorge, Gonalons, Keane y Maffrand, 2003; Vickers, Webster, Wyatt, Dourish y Kennett, 2003), y la tolerancia era más rápida en los animales delgados que en los obesos (Vickers et al. 2003). El descenso del peso mantenido apoya que, además de los efectos anorexígenos, los antagonistas cannabinoides tienen efectos metabólicos. En animales obesos, tras la supresión 
del tratamiento, se producía un efecto rebote con un incremento del peso (Vickers et al. 2003).

\section{Los niveles de endocannabinoides se modifican según la situación de la ingesta alimentaria}

Los cannabinoides endógenos parecen formar parte del sistema de señales que favorecen el inicio de la ingesta. Así en un experimento con animales se objetiva que, en situación de deprivación alimentaria, se incrementan los niveles de 2-AG hipotalámico y de 2-AG y AEA límbicos. Tras la ingesta disminuyen los niveles de 2-AG hipotalámicos. Sin embargo, no hay cambios en dichos niveles en animales saciados o en centros cerebrales no relacionados con la ingesta alimentaria. Igualmente, la administración de 2-AG en el NAc induce la ingesta (Kirkham et al. 2002).

\section{Efecto de los antagonistas cannabinoides en modelos animales de obesidad}

Los experimentos con animales obesos, ya sea obesidad obtenida a través de manipulación genética o inducida por la dieta (DIO, diet-induced obesity), sugieren la presencia de un estado de hiperactividad del sistema cannabinoide en la obesidad. Así, los antagonistas cannabinoides disminuyen la ingesta de forma más eficaz en los animales obesos que en los delgados y disminuyen el peso y el tejido adiposo (Vickers et al. 2003). Además el tratamiento con antagonistas produce una serie de cambios metabólicos favorables, produciendo un descenso de los niveles de leptina, de insulina, de ácidos grasos libres, de colesterol y mejora el estado de resistencia insulínica (Hildebrandt, Kelly-Sullivan y Black, 2003; Ravinet Trillou et al. 2003). El efecto beneficioso sobre el peso se observa en animales emparejados por la cantidad de ingesta alimentaria y el efecto es mayor en animales en ayunas (Ravinet Trillou et al. 2003). Queremos destacar este último hallazgo por sus implicaciones clínicas, es decir, el efecto del rimonabant sobre el peso sería mayor cuando se asocia a una dieta hipocalórica, posiblemente porque en ayunas existe un estado de hiperactividad deI SEC que permite que el rimonabant sea más eficaz.

\section{Los animales carentes del gen del receptor CB1 son más delgados y más resistentes al desarrollo de obesidad}

Los experimentos con animales $\mathrm{KO}$ para el receptor CB1, es decir, carentes de dicho receptor, confirman el papel destacado de dicho receptor en la regulación de la alimentación y en la fisiopatología de la obesidad. Así, dichos animales son más delgados que los con- troles, tienen menos tejido adiposo a pesar de tener la misma ingesta energética, tienen menores niveles de leptina e insulina plasmáticas, presentan menos resistencia a la insulina y resulta de enorme interés destacar que estos animales no desarrollan obesidad inducida por la dieta, lo que indica que el receptor CB1 es clave en el desarrollo de este tipo de obesidad (Ravinet Trillou, Delgorge, Menet, Arnone y Soubrie, 2004).

\section{Estudios en humanos: el consumo de marihuana incrementa el apetito}

Desde hace varias décadas se ha referido que el consumo de cannabis provoca un apetito voraz, con una mayor apetencia por los dulces. Tart (1970) refería que la intoxicación por marihuana producía en los sujetos la apreciación de nuevas cualidades de los alimentos. Hollister (1971) observó que la administración de THC oral en 12 voluntarios en situación de saciedad y en ayuno producía un incremento de la ingesta en 7 de ellos. Abel (1971) sugirió una mayor apetencia por dulces. Greenberg, Kuehnle, Mendelson y Bernstein (1976) observan que el THC fumado aumenta la ingesta y produce un incremento mayor de $2 \mathrm{~kg}$ en las tres semanas del experimento frente al grupo control. Igualmente, Foltin, Brady y Fischman (1986) observan un aumento de las conductas de picar sin que aumente la cantidad de las comidas y, en otro experimento, un incremento de la ingesta de dulces y un aumento mayor del peso de lo esperable por la ingesta calórica (Foltin, Fischman y Byrne, 1988). Mattes, Engelman, Shaw y Elsohly (1994) señalan que, en ciertas condiciones experimentales, el THC incrementa las conductas de picar.

Sin embargo, no está descrito que los consumidores habituales de cannabis tengan un peso superior, lo que sugiere que existe una tolerancia con el tiempo a estos efectos o que haya otras variables importantes que influyen en el peso de los consumidores habituales.

En ensayos clínicos con agonistas cannabinoides en varias patologías se confirma este efecto orexígeno, así aumentan el peso y el tejido graso en pacientes con SIDA (Abrams, Hilton, Leiser, Shade, Elbeik, Aweeka et al. 2002), cáncer (Jatoi, Windschitl, Loprinzi, Sloan, Dakhil, Mailliard et al. 2002) o enfermedad de Alzheimer (Volicer, Stelly, Morris, McLaughlin y Volicer, 1997).

\section{La abstinencia de cannabis produce anorexia}

Varios experimentos en humanos con el consumo de marihuana fumada o vía oral observan como la abstinencia de esta produce anorexia (Haney, Ward, Comer, Foltin y Fischman, 1999a; 1999b). En una revi- 
sión sobre el tema se señala que en 15 de 18 estudios sobre el síndrome de abstinencia de cannabis, la pérdida de apetito es un síntoma de este y, por lo tanto, se propone como criterio diagnóstico de dicho síndrome (Budney, Hughes, Moore y Vandrey, 2004).

\section{El sistema cannabinoide es un elemento crucial en los mecanismos de regulación de la ingesta ali- mentaria}

Estos trabajos indican que el SEC tiene un papel importante en la regulación de la ingesta y del peso y van surgiendo datos que nos orientan sobre los mecanismos que utiliza dicho sistema: por un lado, los cannabinoides tienen un efecto orexígeno central y modulan la acción de múltiples péptidos con papel en la regulación de la ingesta; por otro lado, el SEC tiene un papel en la valoración hedónica de la ingesta y, por último, la función del SEC en la regulación periférica del metabolismo va adquiriendo una enorme relevancia.

Los receptores CB1 están presentes en centros relacionados con la ingesta alimentaria, como núcleos hipotalámicos (Romero, Wenger, De Miguel, Ramos y Fernández-Ruiz, 1998), NAc (Breivogel y Childers, 1998), NTS y núcleo motor dorsal del vago (Derbenev, Stuart y Smith, 2004) y se expresan en los circuitos peptidérgicos hipotalámicos que regulan la ingesta (Horvath, 2003). EI SEC forma parte de los complejos mecanismos que regulan la ingesta alimentaria y así se han descrito interacciones de este sistema con múltiples péptidos con un papel central o periférico en dicha regulación. De estas interacciones hay que destacar que el SEC está presente en el circuito de control de la ingesta regulado por la leptina (Di Marzo, Goparaju, Wang, Liu, Batkai, Jarai et al. 2001).

\section{El sistema cannabinoide modula los efectos refor- zantes de los alimentos}

EI SEC forma parte del sistema de recompensa cerebral y tiene importancia en la biología de la conducta adictiva en general. Respecto a los aspectos motivacionales de la ingesta, el SEC posiblemente está implicado en las dos fases que se describen. Berridge (1996) diferencia la fase incentiva, "wanting" o de apetito, de la fase consumatoria, que denomina "liking".

Los receptores CB1 están localizados a nivel presináptico en las neuronas que liberan dopamina que partiendo del área tegmental ventral (VTA) alcanzan el NAc. Este circuito es clave en los procesos incentivos de la ingesta. Estas neuronas dopaminérgicas liberan 2-AG. En el NAc (en la porción "shell", la más relevante en los procesos incentivos) existe una elevada densidad de receptores CB1 (Lupica, Riegel y Hoffman,
2004). La administración de THC estimula la liberación de dopamina en el NAc (Gardner, 2005).

Diversos experimentos en animales sugieren que los agonistas cannabinoides incrementan el valor incentivo de los alimentos, dado que disminuyen la latencia para la ingesta e induce la ingesta en animales saciados donde la motivación para comer es mínima (Higgs, Williams y Kirkham, 2003). Por el contrario, los antagonistas cannabinoides disminuyen el valor incentivo de los alimentos (Duarte, Alonso, Bichet, Cohen, Soubrie y Thiebot, 2004; Freedland, Sharpe, Samson y Porrino, 2001).

Pero el SEC también media los aspectos orosensoriales de la ingesta. Los agonistas cannabinoides favorecen la toma de sustancias más apetitosas (Arnone et al. 1997; Higgs et al. 2003; Koch y Matthews, 2001; Simiand et al. 1998).

Diversos experimentos en animales que han valorado los efectos en ambas fases, parecen confirmar que el SEC interviene en ambos componentes motivacionales. Así el rimonabant incrementa la latencia para la ingesta y disminuye el número de tomas de alimentos apetitosos (Freedland et al. 2001, ThorntonJones, Vickers y Clifton, 2005).

\section{El sistema cannabinoide regula el metabolismo energético a nivel periférico}

Los datos más recientes van subrayando la creciente importancia del SEC en la regulación periférica de la ingesta y el metabolismo energético. Este papel periférico se apoya, entre otros datos, por el efecto anorexígeno de antagonistas cannabinoides que no atraviesan la BHE, los niveles elevados de AEA en el intestino delgado en ayunas, el efecto anorexígeno del rimonabant por interactuar con la CCK en la periferia o el efecto sobre el apetito de la oleiletanolamina (OEA), un análogo de la anandamida aunque sin efecto sobre los receptores CB1. Además se va describiendo la presencia de receptores cannabinoides en los tejidos periféricos relacionados con el metabolismo energético (tejido adiposo, tubo digestivo, hígado o músculo) y, también está descrita la presencia de receptores CB1 en neuronas vagales.

Se ha sugerido que la AEA puede ser una señal a corto plazo de hambre en la periferia, dado que el ayuno incrementa los niveles de esta en el intestino (Harrold y Williams, 2005).

\section{EI SEC regula el metabolismo lipídico en el tejido adiposo}

Los receptores CB1 están presentes en el tejido adiposo de animales y humanos y los agonistas de este favorecen la lipogénesis (Cota, Marsicano, Tschop, Grubler, Flachskamm, Schubert et al. 2003; 
Lichtman y Cravatt, 2005). Los adipocitos de animales obesos presentan una sobreexpresión de receptores CB1 (Bensaid et al. 2003). Por otro lado, el rimonabant disminuye el tejido adiposo de forma independiente a su efecto anorexígeno. Este antagonista favorece la liberación de adiponectina del tejido adiposo, estimula la oxidación de ácidos grasos libres y disminuye la hiperglucemia y la hiperinsulinemia, más en animales obesos que delgados (Bensaid et al. 2003). El rimonabant estimula la lipolísis por inducción de enzimas que favorecen la oxidación lipídica, restaura la morfología de los adipocitos de los animales obesos y revierte las alteraciones en la expresión génica de estos adipocitos (Jbilo, Ravinet Trillou, Arnone, Buisson, Bribes, Peleraux et al. 2005). Además, el rimonabant inhibe la proliferación de los preadipocitos y favorece la maduración de los adipocitos con lo que disminuye el tejido graso (Gary-Bobo, Elachouri, Scatton, Le Fur, OuryDonat y Bensaid, 2006). Todo esto apoya un papel muy beneficioso de los antagonistas cannabinoides en el metabolismo lipídico de sujetos obesos.

\section{EI SEC modula el metabolismo lipídico en el hígado}

También se ha descrito recientemente la presencia de receptores CB1 en el hígado (Osei-Hyiaman, DePetrillo, Pacher, Liu, Radaeva, Batkai et al. 2005). El hígado es un órgano fundamental en la lipogénesis. Los cannabinoides promueven la lipogénesis hepática y favorecen la esteatosis a través de los receptores CB1 (Kola, Hubina, Tucci, Kirkham, Garcia, Mitchell et al. 2005; Schwabe, 2005). Mientras que los antagonistas como el rimonabant bloquean esos efectos. En animales con obesidad inducida por la dieta (DIO) existe un aumento de la síntesis de ácidos grasos mediados por los receptores CB1 hepáticos. En estos animales se producen niveles elevados de AEA hepática posiblemente por descenso de la actividad de su enzima catabólica, la amidohidrolasa de ácidos grasos (FAAH). Los antagonistas cannabinoides previenen el aumento de densidad de receptores CB1 y el aumento de la síntesis de ácidos grasos hepáticos y estos cambios están ausentes en animales knock-out para el CB1 (Osei-Hyiaman et al. 2005). La regulación de la sintasa de ácidos grasos (FAS) a través de los receptores CB1 se ha propuesto como vía común de los efectos centrales hipotalámicos y periféricos de los cannabinoides (Lichtman y Cravatt, 2005).

\section{EI SEC modula el metabolismo en el músculo}

Los receptores CB1 también se expresan en el músculo estriado. En ratones obesos genéticamente, el rimonabant incrementa el gasto energético, aumenta la captación de glucosa por el músculo y favorece la termogénesis, con lo que mejoran los niveles de glucemia (Liu, Connoley, Wilson y Stock, 2005).
El receptor $C B 1$ y la enzima amidohidrolasa de ácidos grasos (FAAH) están presentes en la mucosa gástrica humana

El equipo de investigación de nuestro hospital ha descrito la presencia de receptores CB1 en el plexo mientérico o plexo de Auerbach del estómago humano relacionado con la motilidad, en el plexo submucoso o plexo de Meissner relacionado con la secreción gástrica y en las células parietales de la mucosa gástrica y de la enzima FAAH en estas últimas células, lo que sugiere un papel en la secreción y en la motilidad gástrica (Pazos, Tolón, Benito, Nuñez, Arias, Gorgojo et al. 2006). La distribución de los receptores CB1 similar a la de la grelina o al péptido similar al glucagon (GLP1, glucagon like peptide 1) y en estructuras neurales modulando transmisión colinérgica y no colinérgica (Adami, Frati, Bertini, Kulkarni-Narla, Brown, de Caro et al. 2002; Storr, Gaffal, Saur, Schusdziarra y Allescher, 2002), sugieren que forman parte de las conexiones entre tubo digestivo y cerebro regulando la conducta alimentaria.

\section{Sistema cannabinoide y trastornos de la conducta alimentaria}

En la etiología de los trastornos de la conducta alimentaria (TCA) intervienen factores genéticos y ambientales. Entre los factores genéticos se han investigado como posibles genes candidatos aquellos que codifican péptidos relacionados con la regulación de la ingesta alimentaria (Kas, Van Elburg, Van Engeland y Adan, 2003). Por lo tanto, el SEC como sistema relevante en dicha regulación es otro candidato para el estudio. Para valorar el papel del SEC en los TCA vamos a hacer referencia a tres aspectos: estudios genéticos, niveles de cannabinoides en pacientes con TCA y uso de agonistas cannabinoides en los TCA.

\section{Estudios genéticos del SEC en los trastornos de la conducta alimentaria}

Estudios de ligamiento genético han identificado al cromosoma 1 como relevante en la genética de los TCA (Grice, Halmi, Fichter, Strober, Woodside, Treasure et al. 2002). En el brazo corto de dicho cromosoma se sitúa el gen que codifica la enzima FAAH, principal enzima catabólica de los endocannabinoides. De este gen se ha descrito un polimorfismo (C385A) que consiste en el cambio de una base de citosina por adenina, lo que conlleva la conversión de un residuo de prolina en treonina en la proteína, lo que la convierte en una enzima menos activa (Chiang, Gerber, Sipe y Cravatt, 2004). Nosotros hemos investigado este polimorfismo en una muestra de 47 pacientes con TCA y 98 controles, sin observar diferencias significativas 
(Arias, Ampuero, Sagredo, Maestro, Almodovar, Sánchez et al. 2007).

Otro gen candidato es el que codifica el receptor CB1, situado en el brazo largo del cromosoma 6, en el que se ha identificado un polimorfismo consistente en la repetición de un triplete de bases AAT de 7 a 15 veces, describiéndose 9 formas alélicas. Un primer estudio en 52 familias de pacientes con TCA observó una asociación de la anorexia nerviosa purgativa con una forma alélica y de la anorexia restrictiva con otro alelo distinto (Siegfried, Kanyas, Latzer, Karni, Bloch, Lerer et al. 2004). En nuestro estudio observamos una falta del alelo 8 y un exceso del alelo 7 en las mujeres con TCA que no alcanzó la significación estadística posiblemente por el pequeño tamaño muestral $(p=0.01)$ (Arias et al. 2007). Por lo tanto, no disponemos aún de datos consistentes que impliquen a factores genéticos relacionados con el SEC en la etiología de los TCA.

Niveles de endocannabinoides en los trastornos de la conducta alimentaria

Un estudio investigó los niveles de AEA y 2-AG en sangre en pacientes con TCA. Se observó que los niveles de AEA estaban elevados en la anorexia nerviosa y en el trastorno por atracones pero no en la bulimia. Los autores sugieren que la AEA está implicada en los aspectos reforzantes de las alteraciones conductuales de la ingesta, pero según esta idea deberían estar alterados dichos niveles también en la bulimia. Además habría que verificar la procedencia de la AEA medida y como refleja los niveles plasmáticos de AEA la funcionalidad central del SEC. De mayor interés es la observación de una correlación negativa entre niveles de AEA y leptina, lo que sugiere un déficit de leptina en la anorexia nerviosa y una insensibilidad a la leptina en el trastorno por atracones que explicaría los niveles elevados de AEA en ambos (Monteleone, Matias, Martiadis, De Petrocellis, Maj y Di Marzo, 2005).

Uso de agonistas cannabinoides en los trastornos de la conducta alimentaria

Sólo disponemos, a nuestro conocimiento, de un ensayo clínico sobre el uso de agonistas en los TCA. Este ensayo, doble ciego y randomizado con la administración de THC en 11 pacientes con anorexia nerviosa, concluyó que el uso de THC era ineficaz y tenía efectos psíquicos adversos (Gross, Ebert, Faden, Goldberg, Kaye, Caine et al. 1983). De cualquier forma, se ha referido que las dosis usadas de THC eran elevadas y estas dosis pueden tener un efecto anorexígeno, posiblemente a través de la liberación de $\mathrm{CRH}$.
En resumen, con la información disponible no podemos decir que el SEC sea relevante en la fisiopatología de los TCA.

\section{Sistema cannabinoide y obesidad}

La obesidad es uno de los principales problemas de salud pública en los países occidentales en la actualidad por su elevada prevalencia. El diagnóstico se realiza considerando simplemente un dato, el índice de masa corporal (IMC). En España, el estudio SEEDO calcula que un $14.5 \%$ de la población tiene un IMC>30 (Aranceta, Pérez, Serra, Ribas, Quiles y Vioque, 2003), en Estados Unidos se calcula que el $30.5 \%$ de la población tiene un IMC> 30 y un 5\% un IMC>40 (Hedley, Ogden, Johnson, Carroll, Curtin y Flegal, 2004).

La obesidad presenta un elevado componente hereditario (Bulik, Sullivan y Kendler, 2003). Las formas monogénicas están descritas pero son raras, siendo considerada como una enfermedad poligénica. Se considera que hay una serie de genes ahorradores que son ventajosos en épocas de escasez de alimentos pero que en el ambiente actual favorecen el desarrollo de obesidad (Bell, Walley y Froguel, 2005).

Dada la relevancia del SEC en la regulación del apetito y del metabolismo energético es un claro sistema candidato al estudio en la obesidad y los datos disponibles demuestran que es un sistema relevante en la fisiopatología de esta. Vamos a hacer referencia a 3 líneas de investigación: estudios genéticos del SEC en obesidad, niveles de endocannabinoides en obesidad y ensayos clínicos con antagonistas cannabinoides en obesidad. Como veremos, todos ellos sugieren que la obesidad podría deberse a un estado de hiperactividad del sistema cannabinoide.

\section{Estudios genéticos del SEC en obesidad}

Se ha descrito la asociación del cromosoma 6 con la obesidad (Loos, Katzmarzyk, Rao, Rice, Leon, Skinner et al. 2003), cromosoma en el cuál, como hemos referido, se encuentra el gen que codifica el receptor CB1. Igualmente, se ha observado una asociación entre el polimorfismo del gen que codifica la enzima FAAH (C385A) y la obesidad (Sipe, Waalen, Gerber y Beutler, 2005). Existía una mayor prevalencia de la forma homocigota mutada AA en obesos que en controles. Esta forma mutada posiblemente implica una menor actividad de la enzima FAAH, una menor inactivación de los endocannabinoides y, por lo tanto, un estado de hiperactividad cannabinoide en obesidad (Sipe et al. 2005).

Nosotros hemos estudiado dicho polimorfismo de la enzima FAAH y el polimorfismo de repetición AATn 
del gen CNR1 en una muestra de 79 obesos mórbidos (media de IMC de 46) y 98 controles normopeso. No obtuvimos diferencias entre los genotipos $A A, A C$ y CC de la enzima FAAH entre obesos y controles, posiblemente debido a que la prevalencia del alelo $A$ era muy baja. Tampoco obtuvimos diferencias entre las distintas formas alélicas del polimorfismo AATn del gen CNR1, aunque cuando se dividieron en genotipos corto/corto, corto/largo y largo/largo siguiendo los criterios de Comings, Muhleman, Gade, Johnson, Verde, Saucier et al. (1997) se observaba el doble de frecuencia del genotipo corto/corto en obesos (15.2\% vs 8.7 $\%$ en controles) $(p=0.18)$. Hay evidencias de que el genotipo corto conlleva una mayor funcionalidad del receptor CB1 (Ampuero, 2005). Además objetivamos una posible interacción entre ambos polimorfismos, de esta forma, la presencia de dos alelos cortos del gen CNR1 y, al menos, un alelo $A$ del gen de la FAAH se asociaba a un mayor riesgo de obesidad mórbida. Entre los sujetos con genotipo AA o AC, un $23.3 \%$ de los obesos tenían polimorfismo corto/corto del gen CNR1 vs un 6.1\% en controles (OR=4.7, IC 95\%: 1-25; $\mathrm{p}=0.05)$, lo que sugiere una mayor hiperactividad del SEC en la obesidad mórbida debido a una mayor actividad funcional del receptor CB1 y una menor actividad de la enzima FAAH (Gorgojo, Arias, Almódovar, Sánchez, Ramos, Alvarez et al. 2006). Las interacciones entre genes en la etiología de la obesidad mórbida se ha descrito previamente (Dong, Li, Geller, Lei, Li, Gorlova et al. 2005).

Niveles de endocannabinoides en tejidos periféricos en la obesidad

Un estudio investigó los niveles plasmáticos de endocannabinoides y la expresión en tejido adiposo del receptor CB1 y de la enzima FAAH en mujeres postmenopáusicas obesas. Se detectaron niveles elevados de AEA y 2-AG plasmáticos y una menor expresión del receptor CB1 y de la enzima FAAH en el tejido adiposo en los obesos respecto al grupo control. La pérdida de peso no afectó a los niveles de endocannabinoides ni a la expresión de CB1 o FAAH, lo que sugiere que habría un estado de hiperactividad del SEC en la obesidad como causa y no consecuencia del aumento de peso y que, posiblemente, el estado de hiperactividad podría ser debido a un descenso de la actividad de la enzima FAAH en la periferia (Engeli, Bohnke, Feldpausch, Gorzelniak, Janke, Batkai et al. 2005).

\section{Sistema cannabinoide y evolución de la obesidad}

Se ha sugerido que el estado de hiperactividad cannabinoide puede explicar las recaídas en la obesidad (Engeli et al. 2005). Nosotros hemos estudiado los polimorfismos genéticos del SEC previamente men- cionados como predictores de respuesta al tratamiento médico y quirúrgico de la obesidad. Observamos que los pacientes homocigotos para el polimorfismo corto del gen CNR1 tenían una menor pérdida ponderal (un 5\% menos de pérdida, IC95\%: 1.3-8.9) tras el tratamiento médico (dieta y fármacos) ajustando por otras variables relevantes que los obesos con polimorfismos largos, sugiriendo que una hiperactividad del sistema cannabinoide puede repercutir en una evolución más desfavorable (Gorgojo et al. 2006).

Por lo tanto, variantes genéticas que pueden implicar una mayor funcionalidad del sistema cannabinoide pueden ser relevantes en la etiología de la obesidad y, a su vez, repercutir en la evolución ponderal de estos pacientes.

\section{Uso de antagonistas cannabinoides en obesidad}

A raíz de los datos mencionados que vinculan al SEC en la etiología de la obesidad se ha desarrollado un amplio programa de desarrollo de un antagonista cannabinoide, el rimonabant. Existen 4 ensayos clínicos con el uso de rimonabant en obesidad, los estudios RIO (Rimonabant in obesity): RIO-Lipids, RIO-North America, RIO-Europe y RIO-Diabetes y 3 ensayos clínicos para el uso en dependencia de nicotina, los estudios STRATUS (Studies with rimonabant and tobacco use) (Boyd y Fremming, 2005). De estos, los cuatro primeros están publicados por lo que los vamos a repasar brevemente. Los datos sugieren que el rimonabant consigue disminuir el peso de los sujetos obesos pero, además, mejora el síndrome metabólico y desciende el consumo de tabaco, por lo que ha llegado a considerarse el "Prozac de la obesidad", con unas previsiones de ventas muy importantes en un futuro próximo (Wadman, 2006).

En el estudio RIO-Europe se incluyeron 1507 sujetos con un IMC>27. Se compararon 5 y $20 \mathrm{mg} / \mathrm{dí}$ de rimonabant versus placebo, junto a dieta hipocalórica. En el análisis realizado a los 12 meses de seguimiento, el grupo de pacientes tratados con 20 mg de rimonabant presentaba una mayor pérdida de peso que el grupo placebo y una mejora en el perfil lipídico que no se explicaba a juicio de los investigadores por la pérdida de peso producida. La pérdida de más de un 10\% de su peso basal, lo que se considera clínicamente relevante, se produjo en un $27.4 \%$ del grupo de rimonabant con $20 \mathrm{mg} / \mathrm{d}$ y en un $7.3 \%$ del grupo placebo. La causa más frecuente de abandonos fue la aparición de depresión pero en todos los grupos de comparación. El uso de 20 mg de rimonabant se asoció con más abandonos por molestias digestivas, cefalea y mareos (Van Gaal, Rissanen, Scheen, Ziegler, Rossner et al. 2005).

El estudio RIO-Lipids (Despres, Golay y Sjostrom, 2005) Ilevado a cabo en 1036 sujetos con un IMC>27, 
comparó igualmente 5 y 20 mg/día de rimonabant versus placebo, junto a dieta hipocalórica, durante 12 meses de seguimiento. Los pacientes tratados con 20 mg/día de rimonabant perdían 6.7 kg más que el grupo placebo, además de presentar una mejora de los niveles de adiponectina, leptina, HDL y triglicéridos. La pérdida de más de un $10 \%$ de su peso basal se produjo en un $32.6 \%$ del grupo de rimonabant con $20 \mathrm{mg} / \mathrm{d}$ y en un 7\% del grupo placebo. Los abandonos más frecuentes fueron por molestias digestivas y alteraciones psiquiátricas (ansiedad y depresión).

En el ensayo RIO-North America se incluyeron 3045 sujetos, con un diseño similar a los anteriores pero al año de seguimiento nuevamente se les volvió a randomizar hasta completar otro año más de evolución. Los resultados son consistentes con los ensayos previos en cuanto a mayor pérdida de peso en el grupo de 20 mg de rimonabant, con una magnitud de efecto similar respecto al grupo placebo (pérdida de $6.3 \mathrm{~kg}$ vs $1.6 \mathrm{~kg}$ ) y similares cambios en el metabolismo lipídico y glucémico que no se explicaban por la cuantía de la pérdida de peso. Tras la segunda randomización, los sujetos que tomaban 20 mg de rimonabant mantenían la pérdida de peso mientras que los asignados al grupo placebo recuperaban el peso perdido. Se produjeron un 6.2\% en el grupo de 20 mg y un $2.3 \%$ en el grupo placebo de abandonos por trastornos psiquiátricos (Pi-Sunyer, Aronne, Heshmati, Devin y Rosenstock, 2006).

El último ensayo clínico publicado es el RIO-Diabetes, que estudia la eficacia de rimonabant en 1047 pacientes obesos con diabetes tipo 2 en tratamiento con antidiabéticos orales. El grupo de 20 mg perdió una media de $5.3 \mathrm{~kg}$ al año de seguimiento, frente a $1.4 \mathrm{~kg}$ en el grupo placebo. Además existía una mejora en la prevalencia del síndrome metabólico y en las cifras de hemoglobina glicosilada. Los efectos adversos fueron ligeramente superiores en el grupo de 20 mg (Scheen, Finer, Hollander, Jensen y Van Gaal, 2006).

Por lo tanto, se puede concluir que el uso de rimonabant produce una pérdida moderada de peso en sujetos obesos, de magnitud similar a otros fármacos antiobesidad (Li, Maglione, Tu, Mojica, Arterburn, Shugarman et al. 2005) pero, además, produce una mayor mejoría del perfil lipídico.

De estos ensayos se desprende que el fármaco habría que administrarse a largo plazo para evitar el riesgo de recaída y que los principales efectos secundarios son los digestivos y las alteraciones psicopatológicas. Estas últimas posiblemente serían el principal inconveniente para el uso de rimonabant en obesidad, si consideramos la elevada prevalencia de trastornos psiquiátricos en sujetos obesos (Arias, Sánchez, Gorgojo, Almódovar, Fernández y Llorente, 2006). Los mecanismos de acción del rimonabant en la obesidad son centrales y periféricos: efecto anorexígeno central, descenso de la motivación para la ingesta, estímulo de señales de saciedad en el tubo digestivo, incremento de la lipólisis y de la liberación de adiponectina en el tejido adiposo y aumento de la captación de glucosa en el músculo (Pagotto y Pasquali, 2005). Otros autores sugieren que los antagonistas cannabinoides normalizarían una señal deficiente de leptina presente en los estados de hiperfagia (Jo, Chen, Chua, Talmage y Role, 2005).

\section{CONCLUSIONES}

EI SEC se constituye como un elemento crucial en la regulación de la ingesta y el metabolismo energético. Los agonistas cannabinoides incrementan el apetito mientras que los antagonistas tienen el efecto opuesto tanto en estudios con animales como con humanos. No existe un mecanismo único para explicar este fenómeno, actuando por mecanismos centrales y, cada vez aparecen como más relevantes, periféricos. Los datos sobre la implicación del SEC en la regulación del metabolismo son consistentes entre ellos, quedan por aclarar los mecanismos bioquímicos implicados. No existe evidencia de que el sistema cannabinoide esté implicado en la etiología de los TCA, aunque hay datos sugerentes de una posible hiperactividad de este sistema en la obesidad, además dicha hiperactividad podría ser un factor pronóstico relevante. De esta forma, los ensayos clínicos con un antagonista cannabinoide como el rimonabant sugieren que se podrá disponer de una nueva arma terapéutica para la obesidad con un perfil novedoso e interesante. Los ensayos clínicos son concordantes en que el uso de rimonabant asociado a una dieta hipocalórica produce una moderada pérdida de peso pero, a diferencia de otros fármacos anti-obesidad, produce una mejora sobreañadida en bastantes parámetros del metabolismo lipídico y glucídico que hacen que sea un fármaco novedoso. Sin embargo, no es la panacea para la obesidad pues se plantean una serie de problemas como son: 1. La pérdida de peso es moderada, habitualmente se produce al inicio del tratamiento, pero hay que mantener un fármaco durante bastantes meses para mantener esa mejora moderada dado que al suprimirlo se recupera el peso. 2. Se produce tolerancia al efecto anorexígeno del fármaco, aunque se mantienen los efectos metabólicos favorables. 3. La presencia de alteraciones psicopatológicas en los pacientes obesos pueden ser un inconveniente para el manejo del fármaco.

Quedan múltiples interrogantes que abren un campo de investigación interesante: si es necesario o no que los antagonistas cannabinoides atraviesen la BHE para el efecto sobre el peso con los consiguien- 
tes posibles efectos adversos centrales, si los antagonistas son más eficaces con dieta hipocalórica o sin ella, si tienen un efecto selectivo sobre los distintos tipos de alimentos, cuál es la eficacia en pacientes obesos con atracones, cuáles son los factores predictores de respuesta a estos fármacos o si es factible el uso de agonistas cannabinoides con pocos efectos psicotomiméticos para la anorexia nerviosa.

\section{REFERENCIAS}

Abel, E.L. (1971). Effects of marihuana on the solution of anagrams, memory and appetite. Nature, 231, 260-261.

Abrams, D.I., Hilton, J.F., Leiser, R.J., Shade, S.B., Elbeik, T.A. y Aweeka, F.T., et al. (2003). Short-term effects of cannabinoids in patients with HIV-1 infection: a randomized, placebo-controlled clinical trial. Annals of Internal Medicine, 139, 258-266.

Adami, M., Frati, P., Bertini, S., Kulkarni-Narla, A., Brown, D.R. y de Caro, G., et al. (2002). Gastric antisecretory role and immunohistochemical localization of cannabinoid receptors in the rat stomach. British Journal of Pharmacology, 135, 1598-1606.

Ampuero, I. (2005). Análisis molecular de los genes del sistema endocannabinoide CNR1 y FAAH en dos condiciones psiquiátricas: alcoholismo y depresión comórbida a la enfermedad de Parkinson. Tesis doctoral. Madrid: Universidad Complutense.

Aranceta, J., Pérez, C., Serra, L., Ribas, L., Quiles, J. y Vioque, J., Grupo colaboreativo para el Estudio de la Obesidad en España (2003). Prevalencia de obesidad en España: resultados del estudio SEEDO 2000. Medicina Clínica, 120, 608-612.

Arias, F., Sánchez, S., Gorgojo, J.J., Almódovar, F., Fernández, S. y Llorente, F. (2006). Diferencias clínicas entre pacientes obesos mórbidos con y sin atracones. Endocrinología y Nutrición, 53, 440-447.

Arias, F., Ampuero, I., Sagredo, O., Maestro, B., Almodovar, F. y Sánchez, S., et al. (aceptado para publicación). Lack of association between polymorphisms in cannabinoid receptor genbe (CNR1) and fatty acid amide hydroxylase gene (FAAH) and eating disorders in a preliminary study. Psychiatric Genetics.

Arnone, M., Maruani, J., Chaperon, F., Thiebot, M.H., Poncelet, M. y Soubrie, P., et al. (1997). Selective inhibition of sucrose and ethanol intake by SR 141716, an antagonist of central cannabinoid (CB1) receptors. Psychopharmacology, 132, 104-106.

Avraham, Y., Ben-Shushan, D., Breuer, A., Zolotarev, O., Okon, A. y Fink, N., et al. (2004). Very low doses of delta 8-THC increase food consumption and alter neurotransmitter levels following weight loss. Pharmacology, Biochemistry and Behavior, 77, 675-684.
Avraham, Y., Menachem, A.B., Okun, A., Zlotarav, O., Abel, N. y Mechoulam, R., et al. (2005). Effects of the endocannabinoid noladin ether on body weight, food consumption, locomotor activity, and cognitive index in mice. Brain Research Bulletin, 65, 117-123.

Bell, C.G., Walley, A.J. y Froguel, P. (2005). The genetics of human obesity. Nature Review Genetics, 6, 221-234.

Bensaid, M., Gary-Bobo, M., Esclangon, A., Maffrand, J.P., Le Fur, G. y Oury-Donat, F., et al. (2003). The cannabinoid CB1 receptor antagonist SR141716 increases Acrp30 mRNA expression in adipose tissue of obese fa/fa rats and in cultured adipocyte cells. Molecular Pharmacology, 63, 908-914.

Berridge, K.C. (1996). Food reward: brain substrates of wanting and liking. Neuroscience and Biobehavioural Reviews, 20, 1-25.

Berthoud, H.R. (2002). Multiple neural systems controlling food intake and body weight. Neuroscience and Biobehavioural Reviews, 26, 393-428.

Boyd, S.T. y Fremming, B.A. (2005). Rimonabant--a selective CB1 antagonist. Annals of Pharmacotherapy, 39, 684-690.

Breivogel, C.S. y Childers, S.R. (1998). The functional neuroanatomy of brain cannabinoid receptors. Neurobiology of Diseases, 5, 417-431.

Broberger, C. (2005). Brain regulation of food intake and appetite: molecules and networks. Journal of Internal Medicine, 258, 301-327.

Budney, A.J., Hughes, J.R., Moore, B.A. y Vandrey, R. (2004). Review of the validity and significance of cannabis withdrawal syndrome. American Journal of Psychiatry, 161, 1967-1977.

Bulik, C.M., Sullivan, P.F. y Kendler, K.S. (2003). Genetic and environmental contributions to obesity and binge eating. International Journal of Eating Disorders, 33, 293-298.

Chiang, K.P., Gerber, A.L., Sipe, J.C. y Cravatt, B.F. (2004). Reduced cellular expression and activity of the P129T mutant of human fatty acid amide hydrolase: evidence for a link between defects in the endocannabinoid system and problem drug use. Human Molecular Genetics, 13, 2113-2119.

Colombo, G., Agabio, R., Diaz, G., Lobina, C., Reali, R. y Gessa, G.L. (1998). Appetite suppression and weight loss after the cannabinoid antagonist SR 141716. Life Sciences, 63, PL113-117.

Comings, D.E., Muhleman, D., Gade, R., Johnson, P., Verde, R. y Saucier, G., et al. (1997). Cannabinoid receptor gene (CNR1): association with i.v. drug use. Molecular Psychiatry, 2, 161-168.

Cota, D., Marsicano, G., Tschop, M., Grubler, Y., Flachskamm, C. y Schubert, M., et al. (2003). The endogenous cannabinoid system affects energy balance via central orexigenic drive and peripheral lipogenesis. Journal of Clinical Investigation, 112, 423-431.

Derbenev, A.V., Stuart, T.C. y Smith, B.N. (2004). Cannabinoids suppress synaptic input to neurones 
of the rat dorsal motor nucleus of the vagus nerve. Journal of Physiology, 559, 923-938.

Despres, J.P., Golay, A. y Sjostrom, L.; Rimonabant in Obesity-Lipids Study Group. (2005). Effects of rimonabant on metabolic risk factors in overweight patients with dyslipidemia. New England Journal of Medicine, 353, 2121-2134.

Di Marzo, V., Goparaju, S.K., Wang, L., Liu, J., Batkai, S. Y Jarai, Z., et al. (2001). Leptin-regulated endocannabinoids are involved in maintaining food intake. Nature, 410, 822-825.

Dong, C., Li, W.D., Geller, F., Lei, L., Li, D. y Gorlova, O.Y., et al. (2005). Possible genomic imprinting of three human obesity-related genetic loci. American Journal of Human Genetic, 76, 427-437.

Duarte, C., Alonso, R., Bichet, N., Cohen, C., Soubrie, P. y Thiebot, M.H. (2004). Blockade by the cannabinoid CB1 receptor antagonist, rimonabant (SR141716), of the potentiation by quinelorane of food-primed reinstatement of food-seeking behavior. Neuropsychopharmacology, 29, 911-920.

Engeli, S., Bohnke, J., Feldpausch, M., Gorzelniak, K., Janke, J. y Batkai, S., et al. (2005). Activation of the peripheral endocannabinoid system in human obesity. Diabetes, 54, 2838-2843.

Foltin, R.W., Brady, J.V. y Fischman, M.W. (1986). Behavioral analysis of marijuana effects on food intake in humans. Pharmacology, Biochemistry and Behavior, 25, 577-582.

Foltin, R.W., Fischman, M.W. y Byrne, M.F. (1988). Effects of smoked marijuana on food intake and body weight of humans living in a residential laboratory. Appetite, 11, 1-14. Freedland, C.S., Poston, J.S. y Porrino, L.J. (2000). Effects of SR141716A, a central cannabinoid receptor antagonist, on food-maintained responding. Pharmacology, Biochemistry and Behavior, 67, 265-270.

Freedland, C.S., Sharpe, A.L., Samson, H.H. y Porrino, L.J. (2001). Effects of SR141716A on ethanol and sucrose self-administration. Alcoholism, Clinical and Experimental Research, 25, 277-282.

Gardner, E.L. (2005). Endocannabinoid signaling system and brain reward: emphasis on dopamine. Pharmacology, Biochemistry and Behavior, 81, 263-284.

Gary-Bobo, M., Elachouri, G., Scatton, B., Le Fur, G., OuryDonat, F. y Bensaid, M. (2006). The cannabinoid CB1 receptor antagonist rimonabant (SR141716) inhibits cell proliferation and increases markers of adipocyte maturation in cultured mouse 3T3 F442A preadipocytes. Molecular Pharmacology, 69, 471-478.

Gomez, R., Navarro, M., Ferrer, B., Trigo, J.M., Bilbao, A. y Del Arco, I., et al. (2002). A peripheral mechanism for CB1 cannabinoid receptor-dependent modulation of feeding. Journal of Neuroscience, 22, 9612-9617.

Gorgojo, J.J., Arias, F., Almódovar, F., Sánchez, S., Ramos, J.A. y Alvarez, M., et al. (2006). Sistema cannabinoide y regulación de la conducta alimentaria: II. El polimorfismo corto (AAT)n del gen del receptor cannabinoide tipo 1 (CNR1) predice una menor pérdida de peso tras tratamiento médico o quirúrgico en pacientes con obesidad mórbida. Póster presentado al XXXIII Congreso Socidrogalcohol. Cáceres.

Greenberg, I., Kuehnle, J., Mendelson, J.H. y Bernstein, J.G. (1976). Effects of marihuana use on body weight and caloric intake in humans. Psychopharmacology, 49, 79-84.

Grice, D.E., Halmi, K.A., Fichter, M.M., Strober, M., Woodside, D.B. y Treasure, J.T., et al. (2002). Evidence for a susceptibility gene for anorexia nervosa on chromosome 1. American Journal of Human Genetic, 70, 787-792.

Grinspoon, L. y Bakalar, J.B. (1993). Marihuana. The forbidden medicine. London: Yale University Press.

Gross, H., Ebert, M.H., Faden, V.B., Goldberg, S.C., Kaye, W.H. y Caine, E.D., et al. (1983). A double-blind trial of delta 9-tetrahydrocannabinol in primary anorexia nervosa. Journal of Clinical Psychopharmacology, 3, 165-171.

Hao, S., Avraham, Y., Mechoulam, R. y Berry, E.M. (2000). Low dose anandamide affects food intake, cognitive function, neurotransmitter and corticosterone levels in diet-restricted mice. European Journal of Pharmacology, 392, 147-156.

Haney, M., Ward, A.S., Comer, S.D., Foltin, R.W. y Fischman, M.W. (1999a). Abstinence symptoms following smoked marijuana in humans. Psychopharmacology, 141, 395-404.

Haney, M., Ward, A.S., Comer, S.D., Foltin, R.W. y Fischman, M.W. (1999b). Abstinence symptoms following oral THC administration to humans. Psychopharmacology, 141, 385-394.

Harrold, J.A. y Williams, G. (2006). Melanocortin-4 receptors, beta-MSH and leptin: key elements in the satiety pathway. Peptides, 27, 365-371.

Hedley, A.A., Ogden, C.L., Johnson, C.L., Carroll, M.D., Curtin, L.R. y Flegal, K.M. (2004). Prevalence of overweight and obesity among US children, adolescents, and adults, 1999-2002. Journal of American Medical Association, 291, 2847-2850.

Higgs, S., Williams, C.M. y Kirkham, T.C. (2003). Cannabinoid influences on palatability: microstructural analysis of sucrose drinking after delta(9)-tetrahydrocannabinol, anandamide, 2-arachidonoyl glycerol and SR141716. Psychopharmacology, 165, 370-377.

Hildebrandt, A.L., Kelly-Sullivan, D.M. y Black, S.C. (2003). Antiobesity effects of chronic cannabinoid CB1 receptor antagonist treatment in diet-induced obese mice. European Journal of Pharmacology, 462, 125-132.

Hollister, L.E. (1971). Hunger and appetite after single doses of marihuana, alcohol, and dextroamphetamine. Clinical Pharmacology and Therapeutics, 12, 44-49.

Hollister, L. (2001). Marijuana (cannabis) as medicine. Journal of Cannabis Therapeutics, 1, 5-28.

Horvath, T.L. (2003). Endocannabinoids and the regulation of body fat: the smoke is clearing. Journal of Clinical Investigation, 112, 323-326. 
Jamshidi, N. y Taylor, D.A. (2001). Anandamide administration into the ventromedial hypothalamus stimulates appetite in rats. British Journal of Pharmacology, 134, 1151-1154.

Jatoi, A., Windschitl, H.E., Loprinzi, C.L., Sloan, J.A., Dakhil, S.R. y Mailliard, J.A., et al. (2002). Dronabinol versus megestrol acetate versus combination therapy for cancer-associated anorexia: a North Central Cancer Treatment Group study. Journal of Clinical Oncology, 20, 567-573

Jbilo, O., Ravinet-Trillou, C., Arnone, M., Buisson, I., Bribes, E. y Peleraux, A., et al. (2005). The CB1 receptor antagonist rimonabant reverses the diet-induced obesity phenotype through the regulation of lipolysis and energy balance. FASEB Journal, 19, 1567-1569.

Jo, Y.H., Chen, Y.J., Chua, S.C. Jr, Talmage, D.A. y Role, L.W. (2005). Integration of endocannabinoid and leptin signaling in an appetite-related neural circuit. Neuron, 48, 1055-1066.

Kas, M.J., Van Elburg, A.A., Van Engeland, H. y Adan, R.A. (2003). Refinement of behavioural traits in animals for the genetic dissection of eating disorders. European Journal of Pharmacology, 480, 13-20.

Kirkham, T.C., Williams, C.M., Fezza, F. y Di Marzo, V. (2002). Endocannabinoid levels in rat limbic forebrain and hypothalamus in relation to fasting, feeding and satiation: stimulation of eating by 2-arachidonoyl glycerol. British Journal of Pharmacology, 136, 550-557.

Kirkham, T.C. (2005). Endocannabinoids in the regulation of appetite and body weight. Behavioral Pharmacology, 16, 297-313.

Koch, J.F. y Werner, N.A. (2000). Effects of the cannabinoid antagonists AM 630 and AM 281 on deprivationinduced food intake in Lewis rats. Social Neuroscience Abstracts, 26, 569 .

Koch, J.E. y Matthews, S.M. (2001). Delta9tetrahydrocannabinol stimulates palatable food intake in Lewis rats: effects of peripheral and central administration. Nutritional Neuroscience, 4, 179-187.

Kola, B., Hubina, E., Tucci, S.A., Kirkham, T.C., Garcia, E.A. y Mitchell, S.E., et al. (2005). Cannabinoids and ghrelin have both central and peripheral metabolic and cardiac effects via AMP-activated protein kinase. Journal of Biological Chemistry, 280, 25196-25201.

Lichtman, A.H. y Cravatt, B.F. (2005). Food for thought: endocannabinoid modulation of lipogenesis. Journal of Clinical Investigation, 115, 1130-1133.

Li, Z., Maglione, M., Tu, W., Mojica, W., Arterburn, D. y Shugarman, L.R., et al. (2005). Meta-analysis: pharmacologic treatment of obesity. Annals of Internal Medicine, 142, 532-546.

Liu, Y.L., Connoley, I.P., Wilson, C.A. y Stock, M.J. (2005). Effects of the cannabinoid CB1 receptor antagonist SR141716 on oxygen consumption and soleus muscle glucose uptake in Lep ob /Lep ob mice. International Journal of Obesity, 29, 183-187.
Loos, R.J., Katzmarzyk, P.T., Rao, D.C., Rice, T., Leon, A.S. y Skinner, J.S., et al., HERITAGE Family Study. (2003). Genome-wide linkage scan for the metabolic syndrome in the HERITAGE Family Study. Journal of Clinical Endocrinology and Metabolism, 88, 5935-5943.

Lupica, C.R., Riegel, A.C. y Hoffman, A.F. (2004). Marijuana and cannabinoid regulation of brain reward circuits. British Journal of Pharmacology, 143, 227-234.

Martínez-Gonzalez, D., Bonilla-Jaime, H., Morales-Otal, A., Henriksen, S.J., Velázquez-Moctezuma, J. y PrósperoGarcía, O. (2004). Oleamide and anandamide effects on food intake and sexual behavior of rats. Neuroscience Letters, 364, 1-6.

Mattes, R.D., Engelman, K., Shaw, L.M. y Elsohly, M.A. (1994). Cannabinoids and appetite stimulation. Pharmacology, Biochemistry and Behaviour, 49, 187-195.

McLaughlin, P.J., Winston, K., Swezey, L., Wisniecki, A., Aberman, J. y Tardif, D.J., et al. (2003). The cannabinoid CB1 antagonists SR 141716A and AM 251 suppress food intake and food-reinforced behavior in a variety of tasks in rats. Behavioral Pharmacology, 14, 583-588.

Miller, C.C., Murray, T.F., Freeman, K.G. y Edwards, G.L. (2004). Cannabinoid agonist, CP 55,940, facilitates intake of palatable foods when injected into the hindbrain. Physiology and Behavior, 80, 611-616.

Monteleone, P., Matias, I., Martiadis, V., De Petrocellis, L., Maj, M. y Di Marzo, V. (2005). Blood levels of the endocannabinoid anandamide are increased in anorexia nervosa and in binge-eating disorder, but not in bulimia nervosa. Neuropsychopharmacology, 30, 1216-1221.

Osei-Hyiaman, D., DePetrillo, M., Pacher, P., Liu, J., Radaeva, S. y Batkai, S., et al. (2005). Endocannabinoid activation at hepatic $\mathrm{CB} 1$ receptors stimulates fatty acid synthesis and contributes to diet-induced obesity. Journal of Clinical Investigation, 115, 1298-1305.

Pagotto, U. y Pasquali, R. (2005). Fighting obesity and associated risk factors by antagonising cannabinoid type 1 receptors. Lancet, 365, 1363-1364.

Pagotto, U. y Pasquali, R. (2006). Endocannabinoids and energy metabolism. Journal of Endocrinological Investigation, 29(Suppl), 66-76.

Pazos, R., Tolón, R.M., Benito, C., Nuñez, E., Arias, F. y Gorgojo, J.J., et al. (2006). Sistema cannabinoide y regulación de la conducta alimentaria: III. El receptor para cannabinoides CB1 y la enzima hidrolasa de ácidos grasos (FAAH) están presentes en la mucosa gástrica humana. Póster presentado a XXXIII Congreso Socidrogalcohol. Cáceres.

Pi-Sunyer, F.X., Aronne, L.J., Heshmati, H.M., Devin, J. y Rosenstock, J., RIO-North America Study Group. (2006). Effect of rimonabant, a cannabinoid-1 receptor blocker, on weight and cardiometabolic risk factors in overweight or obese patients: RIO-North America: a randomized controlled trial. Journal of American Medical Association, 295, 761-775. 
Ramos, J.A. y Fernández, J. (2000). Uso de los cannabinoides a través de la historia. Adicciones, 12 (supl. 2), 19-30.

Ravinet Trillou, C., Arnone, M., Delgorge, C., Gonalons, N., Keane, P. y Maffrand, J.P., et al. (2003). Anti-obesity effect of SR141716, a CB1 receptor antagonist, in dietinduced obese mice. American Journal of Physiology. Regulatory, Integrative and Comparative Physiology, 284, R345-353.

Ravinet Trillou, C., Delgorge, C., Menet, C., Arnone, M. y Soubrie, P. (2004). CB1 cannabinoid receptor knockout in mice leads to leanness, resistance to diet-induced obesity and enhanced leptin sensitivity. International Journal of Obesity and Related Metabolical Disorders, 28, 640-648.

Romero, J., Wenger, T., De Miguel, R., Ramos, J.A. y Fernández-Ruiz, J.J. (1998). Cannabinoid receptor binding did not vary in several hypothalamic nuclei after hypothalamic deafferentation. Life Sciences, 63, 351-356.

Rowland, N.E., Mukherjee, M. y Robertson, K. (2001). Effects of the cannabinoid receptor antagonist SR141716, alone and in combination with dexfenfluramine or naloxone, on food intake in rats. Psychopharmacology, 159, 111-116.

Scheen, A.J., Finer, N., Hollander, P., Jensen, M.D., Van Gaal, L.F.; RIO-Diabetes Study Group. (2006) Efficacy and tolerability of rimonabant in overweight or obese patients with type 2 diabetes: a randomised controlled study. Lancet, 368, 1660-1672.

Schwabe, R.F. (2005). Endocannabinoids promote hepatic lipogenesis and steatosis through CB1 receptors. Hepatology, 42, 959-961.

Siegfried, Z., Kanyas, K., Latzer, Y., Karni, O., Bloch, M. y Lerer, B., et al. (2004). Association study of cannabinoid receptor gene (CNR1) alleles and anorexia nervosa: differences between restricting and binging/purging subtypes. American Journal of Medical Genetics. Part B, Neuropsychiatric Genetics, 125, 126-130.

Simiand, J., Keane, M., Keane, P.E. y Soubrie, P. (1998). SR 141716, a CB1 cannabinoid receptor antagonist, selectively reduces sweet food intake in marmoset. Behavioral Pharmacology, 9, 179-181.

Sipe, J.C., Waalen, J., Gerber, A. y Beutler, E. (2005). Overweight and obesity associated with a missense polymorphism in fatty acid amide hydrolase (FAAH). International Journal of Obesity, 29, 755-759.

Stellar, E. (1954). The physiology of motivation. Psychological Reviews, 61, 5-22.

Storr, M., Gaffal, E., Saur, D., Schusdziarra, V. y Allescher, H.D. (2002). Effect of cannabinoids on neural transmission in rat gastric fundus. Canadian Journal of Physiology and Pharmacology, 80, 67-76.

Tart, C.T. (1970). Marijuana intoxication common experiences. Nature, 226, 701-704.

Thornton-Jones, Z.D., Vickers, S.P. y Clifton, P.G. (2005). The cannabinoid CB1 receptor antagonist SR141716A reduces appetitive and consummatory responses for food. Psychopharmacology, 179, 452-460.

Van Gaal, L.F., Rissanen, A.M., Scheen, A.J., Ziegler, O. y Rossner, S., RIO-Europe Study Group. (2005). Effects of the cannabinoid-1 receptor blocker rimonabant on weight reduction and cardiovascular risk factors in overweight patients: 1-year experience from the RIOEurope study. Lancet, 365, 1389-1397.

Verty, A.N., McGregor, I.S. y Mallet, P.E. (2004). Consumption of high carbohydrate, high fat, and normal chow is equally suppressed by a cannabinoid receptor antagonist in non-deprived rats. Neuroscience Letters, 354, 217-220.

Vickers, S.P., Webster, L.J., Wyatt, A., Dourish, C.T. y Kennett, G.A. (2003). Preferential effects of the cannabinoid CB1 receptor antagonist, SR 141716, on food intake and body weight gain of obese (fa/fa) compared to lean Zucker rats. Psychopharmacology, 167, 103-111.

Volicer, L., Stelly, M., Morris, J., McLaughlin, J. y Volicer, B.J. (1997). Effects of dronabinol on anorexia and disturbed behavior in patients with Alzheimer's disease. International Journal of Geriatric Psychiatry, 12, 913-919.

Wadman, M. (2006). Rimonabant adds appetizing choice to slim obesity market. Nature Medicine, 12, 27.

Ward, S.J. y Dykstra, L.A. (2005). The role of CB1 receptors in sweet versus fat reinforcement: effect of $\mathrm{CB} 1$ receptor deletion, $\mathrm{CB} 1$ receptor antagonism (SR141716A) and CB1 receptor agonism (CP-55940). Behavioural Pharmacology, 16, 381-388.

Wiley, J.L., Burston, J.J., Leggett, D.C., Alekseeva, O.O., Razdan, R.K. y Mahadevan, A., et al. (2005). CB1 cannabinoid receptor-mediated modulation of food intake in mice. British Journal of Pharmacology, 145, 293-300.

Williams, C.M., Rogers, P.J. y Kirkham, T.C. (1998). Hyperphagia in pre-fed rats following oral delta9-THC. Physiology and Behavior, 65, 343-346.

Williams, C.M. y Kirkham, T.C. (1999). Anandamide induces overeating: mediation by central cannabinoid (CB1) receptors. Psychopharmacology, 143, 315-317.

Williams, C.M. y Kirkham, T.C. (2002). Observational analysis of feeding induced by Delta9-THC and anandamide. Physiology and Behavior, 76, 241-250.

Wynne, K., Stanley, S., McGowan, B. y Bloom, S. (2005). Appetite control. Journal of Endocrinology, 184, 291-318. 
